Won G. Ng · Yan-Kang Xu · Francine R. Kaufman

George N. Donnell · Jon Wolff · Richard J. Allen

Sriveda Koritala $\cdot$ Juergen K. V. Reichardt

\title{
Biochemical and molecular studies of 132 patients with galactosemia
}

Received: 18 January 1994 / Revised: 12 April 1994

\begin{abstract}
We evaluated 132 galactosemia patients for the Q188R (glutamine-188 to arginine) mutation in the human galactose-1-phosphate uridyltransferase (GALT) gene and for GALT activity in their hemolysates by a sensitive radioisotopic method. In those without any detectable GALT activity (GG), the Q188R mutation constituted 67\% of the alleles. In patients with detectable GALT activity (GV), only $16 \%$ of the alleles were accounted for by Q188R. In all patients who were homozygous for the Q188R mutation, no erythrocyte GALT activity could be demonstrated. There was an extensive variation in the amount of detectable GALT activity ranging from $0.1 \%$ to $5 \%$ of the normal values among the GV patients. There was a difference in the frequency of Q188R mutation in the GALT alleles among patients belonging to different racial and ethnic groups. In Caucasian and Hispanic patients, the frequency was not far different $(64 \%$ and $58 \%$, respectively). On the other hand, only $12 \%$ of the GALT alleles with Q188R were found in African-American patients.
\end{abstract}

W. G. Ng (西) · Y.-K. Xu · F. R. Kaufman · G. N. Donnell Childrens Hospital Los Angeles and Department of Pediatrics, University of Southern California School of Medicine, Los Angeles, CA 90027 , USA

J. Wolff

Waisman Center, Department of Pediatrics,

University of Wisconsin, Madison, WI 53706, USA

R. J. Allen

Pediatric Neurology Section, Department of Pediatrics and Neurology,

University of Michigan Medical Center,

Ann Arbor, MI 48 109, USA

J. K. V. Reichardt

Institute for Genetic Medicine,

University of Southern California School of Medicine, Los Angeles, CA 90033, USA

J. K. V. Reichardt · S. Koritala

Department of Biochemistry and Molecular Biology,

University of Southern California School of Medicine,

Los Angeles, CA 90033, USA

\section{Introduction}

Galactosemia (McKusick no. 230400) is an inborn error of galactose metabolism due to galactose-1-phosphate uridyltransferase (GALT) enzyme deficiency (Segal 1989). Clinical and biochemical heterogeneity among galactosemia patients was described in the early 1960s (Hsia and Walker 1961; $\mathrm{Ng}$ et al. 1964). An extensive survey of the clinical and biochemical findings in 350 cases was reported (Waggoner et al. 1990). In 1965 some African-American patients were found to have the capacity, albeit small, to metabolize galactose (Segal et al. 1965). Besides the African-American variant, others such as the isolated cases of the Rennes (Schapira and Kaplan 1969), Indiana (Chacko et al. 1971) and Chicago I (Chacko et al. 1977) variants were described. A recent study of 358 patients suggested that there was a correlation between the severity of the clinical presentation and the degree of enzyme deficiency and that this also was reflected in the erythrocyte galactose-1phosphate (Gal-1-P) and uridine diphosphate galactose (UDPGal) levels (Ng et al. 1991).

The cloning and characterization of the cDNA encoding human galactose-1-phosphate uridyltransferase by Reichardt and Berg (1988) has provided a means of studying the nature of the gene defect in galactosemia and its clinical expression. The entire gene for GALT was sequenced (Leslie et al. 1992), and a number of mutations among galactosemia patients have been uncovered (Reichardt 1992). The most common mutation among galactosemia patients appears to be the Q188R (glutamine-188 to arginine) in exon 6 (Reichardt et al. 1991; Elsas et al. 1993). It has recently been suggested that there was a higher frequency of Q188R homozygosity in patients with a poor outcome (Elsas et al. 1992).

This study presents biochemical findings and Q188R mutation analysis for 132 patients. 
Table 1 Demographic data of 132 patients (NBS, Newborn screening)

\begin{tabular}{ll}
\hline Sex: & 79 females, 53 males \\
Initial suspicion: & NBS (70), clinical (47), siblings (15) \\
Age distribution: & $0-5$ years (54), 6-17 years (36), \\
& $>17$ years (42) \\
Racial and ethnic & Caucasian (106), African American (12) \\
background: & Hispanic (9), African American-Caucasian \\
& (1), African American-Hispanic (1), \\
& Hispanic-Native American (1), Native \\
& American (2) \\
Regional distribution: & West (68), Midwest (34), East (17), South \\
& (10), Canada (3)
\end{tabular}

\section{Materials and methods}

Patients

The total number of patients studied was 132 and the demographic data on their samples is shown in Table 1. All had erythrocyte galactose-1-phosphate uridyltransferase (GALT) assays and DNA Q188R mutation analysis on blood samples collected from filter paper spots. In addition, a number of patients had uridine diphosphate glucose (UDPGlc) and uridine diphosphate galactose measurements made on their erythrocytes by an enzyme coupling method.

\section{Preparation of blood samples}

For GALT assay and UDPGlc and UDPGal measurements, blood was washed three times with physiological saline $(0.9 \mathrm{~g} \% \mathrm{NaCl})$ after removal of plasma and buffy coat. Red blood cells were immediately frozen until analysis. For DNA analysis, whole blood was received in either sodium heparinized or EDTA anticoagulant tubes. Blood was applied onto filter paper spots, air dried, and then stored frozen until analysis.

\section{GALT assay}

The multiple point radioisotopic assay for GALT activity has been described previously ( $\mathrm{Ng}$ et al. 1991), and the details of the assay will be reported in a future publication (W. G. Ng, in preparation). In essence, a $50 \%$ hemolysate was prepared from $0.2-0.3 \mathrm{ml}$ of frozen red blood cells by addition of an equal volume of distilled water. Then $0.2-0.5 \mathrm{ml}$ of the $50 \%$ hemolysate was dialyzed against $1 \mathrm{l}$ of GEM buffer, which was made up of $0.1 \mathrm{M}$ glycine, $0.001 M$ EDTA, and $0.003 M \beta$-mercaptoethanol, $\mathrm{pH} 8.0$ at $4^{\circ} \mathrm{C}$ overnight. Then $20 \mu \mathrm{l}$ of dialyzed hemolysate was incubated with $100 \mu M$ of highly labeled ${ }^{14} \mathrm{C}-\mathrm{Gal}-1-\mathrm{P}$ (sp. act. of $5 \mathrm{mCi} / \mathrm{mM}$ ) from NEN (Dupont, Mass.) and $80 \mu \mathrm{M}$ UDPGlc in a glycine-dithiothreitol buffer, $\mathrm{pH} 8.7$ with a total volume of $100 \mu \mathrm{l}$ for 10,30 , and 60 $\mathrm{min}$. At the end of each incubation period, $10 \mu \mathrm{l}$ aliquots were spotted on DEAE-8 1 paper strips and chromatographed in $0.05 \mathrm{M}$ $\mathrm{LiCl}$. Radioactivities in sections corresponding to UDPhexose (UDPGal) and Gal-1-P were determined by liquid scintillation counting. Galactose-1-phosphate uridyltransferase (GALT) activity was expressed as $\mu \mathrm{mol} / \mathrm{h} / \mathrm{g} \mathrm{Hb}$. The sensitivity of the assay is at about $0.1 \%$ of normal activity. For a blood sample from normal individuals, a $1 \%$ hemolysate should be used because the assay is suitable only for low GALT activity; higher activity would result in rapid disappearance of the substrates.

\section{Erythrocyte UDPGIc and UDPGal measurements}

The levels of these nucleotide sugars were determined in an aqueous boiled extract by a modification of the procedure originally de- scribed by $\mathrm{Ng}$ et al. (1989). The modifications included correction of reagent blanks, which might give fluorescent readings, and omission of cysteine, which was found to be unnecessary. The UDPGlc dehydrogenase supplied from Sigma (U5500) and Boehringer Mannheim was used at $0.00125 \mathrm{U}$ per incubation.

Polymerase chain reaction (PCR) assay

A $1-\mathrm{mm}^{2}$ sample from a blood spot or $0.1 \mu \mathrm{g}$ genomic DNA was amplified in a $100 \mu \mathrm{l}$ reaction with $0.5 \mu \mathrm{g}$ of each primer, sense: 6-5', CTGTTTCCACAGGGTGTGG and anti-sense: 6-3', GCTGGCTCAGACTCAGCC, synthesized on a Beckman Oligo 1(0)(; (Fullerton, Calif.); amplifying all of exon 6 in the nomenclature of Leslie et al. (1992) as follows: after an initial denaturation at $97^{\circ} \mathrm{C}$ for $5 \mathrm{~min}$, primers were annealed for $10 \mathrm{~s}$ at $50^{\circ} \mathrm{C}$ and $1.5 \mathrm{U} T a q$ DNA polymerase (Promega, Madison, Wis.) were added and the products were then extended for $5 \mathrm{~min}$ at $72^{\circ} \mathrm{C}$. Next 40 cycles consisting of denaturation for $1 \mathrm{~min}$ at $94^{\circ} \mathrm{C}$, annealing for $10 \mathrm{~s}$ at $57^{\circ} \mathrm{C}$, and extension for $40 \mathrm{~s}$ at $72^{\circ} \mathrm{C}$ were performed in an Ericomp Twinblock (San Diego, Calif.). Finally, the PCR products were blunted for $5 \mathrm{~min}$ at $72^{\circ} \mathrm{C}$. Successful amplification was verified on a 4\% NuSieve 3:1 analytical gel (FMC, Rockland, Me.).

\section{Hpall assay of Q188R}

A $20-\mu \mathrm{l}$ sample of the $\mathrm{PCR}$ reaction was mixed with $2 \mu \mathrm{l}$ of buffer A (Promega), and $2 \mathrm{U}$ of Hpall (Promega) were added. PCR products were digested overnight at $37^{\circ} \mathrm{C}$ and then analyzed on a $4 \%$ NuSieve 3:1 agarose gel (FMC). The size of the PCR product was $185 \mathrm{bp}$. After the digestion, Q188R mutation resulted in two fragments, $135 \mathrm{bp}$ and $50 \mathrm{bp}$.

\section{Results}

Patients with galactosemia have been classified into two groups: GG (patients without detectable erythrocyte GALT activity) and GV (patients with detectable erythrocyte GALT activity) based on the presence and absence of detectable GALT activity in erythrocytes as determined by the multipoint radioisotopic assay, ( $\mathrm{Ng}$ et al. 1991). Erythrocyte GALT activity in 132 patients in this study was compared with the results obtained by Q188R mutation analysis. The findings are summarized in Table 2. There were 113 patients in the GG group and 19 patients in GV group. In the GG group, 56 patients were homozygous, 40 patients heterozyous, and 17 patients negative for the Q188R mutation. This constituted $67 \%$ of the GALT alleles with the Q188R mutation. In the GV group, 6 patients were heterozygous and 13 patients were negative for the Q188R mutation. Thus, in this group, only $16 \%$ of the GALT alleles had the Q188R mutation. Taken as a whole (GG and GV), 56 patients were homozygous for the Q188R mutation, 46 heterozygous, and 30 did not exhibit the mutation. This yielded $60 \%$ (158/264) of GALT alleles with Q188R mutation. An interesting observation was that all patients homozygous for the Q188R mutation had no detectable GALT activity and none of the patients with detectable GALT activity were homozygous for the Q188R mutation.

Figure 1 shows the spectrum of erythrocyte GALT activity detected for $19 \mathrm{GV}$ patients: 12 were Caucasians, 4 were African Americans, and 3 were Native Americans. 
Table 2 Correlation of Q188R mutation with galactose-1-phosphate uridyltransferase (GALT) activity

\begin{tabular}{lrrll}
\hline Group $^{\mathrm{a}}$ & $+/+$ & $+/-$ & $-1-$ & $\%+$ alleles \\
\hline GG (113) & 56 & 40 & 17 & 67 \\
GV (19) & 0 & 6 & 13 & 16 \\
& 56 & 46 & 30 & 60 \\
\hline
\end{tabular}

a GG, patients without any detectable GALT activity; GV, patients with detectable GALT activity; affected sibs are included

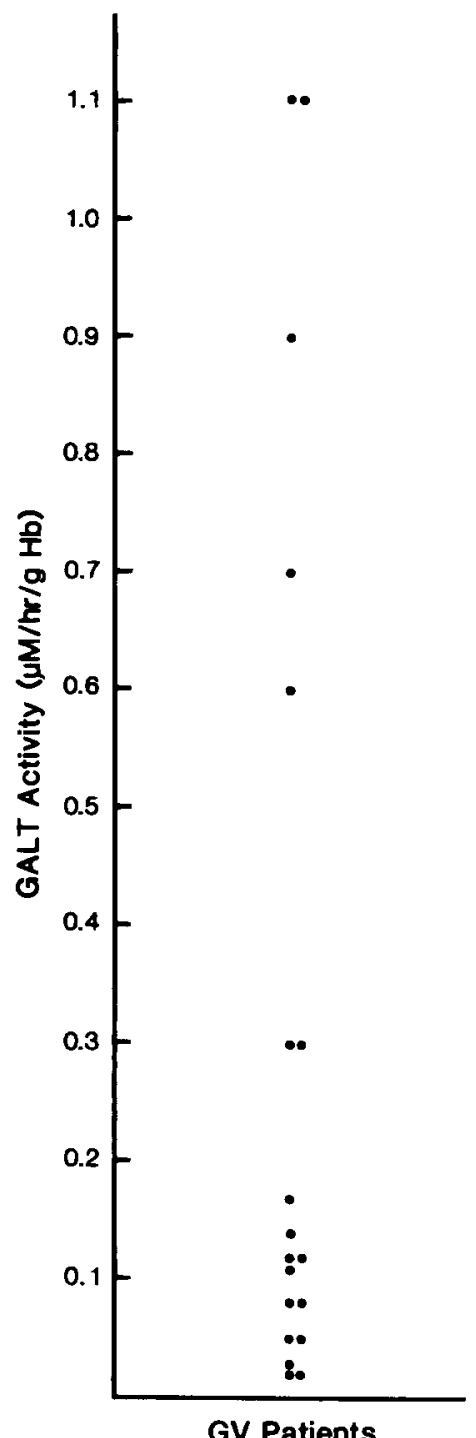

Fig. 1 Spectrum of erythrocyte GALT activity found among 19 GV patients

Of these, 16 patients were identified by newborn screening programs and 3 were initially suspected based on clinical grounds. Of the 16 patients identified by newborn screening, 1 exhibited hepatomegaly and 1 , jaundice. In these 2 patients, who were both African Americans, the GALT activity was low: 0.02 and 0.08 units, respectively (normal $\geq 20$ units). The remaining 14 were asymptomatic, Two of these patients were Native American sib-
Table 3 Q188R analysis of galactosemia patients according to different racial and ethnic background. Affected sibs are excluded

\begin{tabular}{lrrrl}
\hline Group & $+/+$ & $+/-$ & \multicolumn{1}{c}{$-1-$} & $\%+$ alleles \\
\hline Caucasian & 44 & 34 & 17 & 64 \\
African American & 1 & 2 & 9 & 12 \\
Hispanic & 2 & 3 & 1 & 58 \\
African American Hispanic & & 1 & & - \\
African American-Caucasian & & & 1 & - \\
Hispanic-Native American & & 1 & 1 & - \\
Native American & & 1 & & - \\
\hline & 47 & 41 & 29 & 58
\end{tabular}

lings with erythrocyte GALT activities of 0.7 and 0.9 units. One other patient, of Native American and Hispanic descent, also exhibited substantial residual activity equal to 1.1 units. Among the three patients with detectable GALT activity identified on the basis of clinical suspicion, two females had classical clinical onset in the neonatal period. Both of them are now of adult age and continue to exhibit normal ovarian function. The third patient, now a 12-year-old male, was found to have lenticular cataracts as a child but was not suspected of having galactosemia until his younger brother was diagnosed on newborn galactosemia screening. All of the three patients were Caucasians, and their GALT activities were 0.02 , 0.03 , and 0.14 units, respectively.

Table 3 summarizes the results of Q188R mutation analysis on galactosemia patients according to their racial and ethnic background. Data on only index cases were included, while data from affected sibling were excluded from the table. In the Caucasian group, mostly of European origin, the percentage of alleles with Q188R mutation was $64 \%$, and that for the small number of samples in the Hispanic group was similar (58\%). Most of the Hispanic patients came from Mexico and can be classified into the Caucasian group. On the other hand, the frequency of the Q188R mutation in African Americans was considerably lower (12\%). Excluding affected siblings, about $58 \%$ of the GALT alleles in the 117 patients studied had the Q188R mutation.

UDPGlc and UDPGal levels in the erythrocytes were measured by an enzymatic method in 72 of the 132 galactosemic patients in this study (64 GG and $8 \mathrm{GV}$ ). The levels of UDPGlc in GG, GV, and the controls are similar: normals (40) : $21.3 \pm 3.9$, GG (64) : $19 \pm 4.5$, and GV (8): $18.4 \pm 2.1 \mu \mathrm{mol} / 100 \mathrm{~g} \mathrm{Hb}$. However, for UDPGal, all 64 GG patients had lower values than the controls and normal values in all $8 \mathrm{GV}$ patients: normals (40): $8.4 \pm 1.0$, GG (64): $2.5 \pm 0.8$, and GV (8) $8.2 \pm 0.8 \mu \mathrm{mol} / 100 \mathrm{~g} \mathrm{Hb}$. The absolute values by our enzymatic method were higher than those found by HPLC analysis (Palmieri et al. 1991) including our work (Xu et al. 1993). Nevertheless, the findings of lower values of UDPGal in GG and normal values in GV patients were consistent by both methods in our laboratory. 


\section{Discussion}

The successful cloning of the cDNA for human galactose-1-phosphate uridyltransferase was accomplished by Reichardt and Berg (1988). Subsequently, the sequence of the cDNA was revised by Flach et al. (1990), and the entire gene for the enzyme has been sequenced by Leslie et al. (1992).

A number of mutations in the GALT gene have been identified, and the most common of these among galactosemia patients is Q188R in exon 6 (Reichardt et al. 1991; Elsas et al. 1993). Our present study of 132 patients confirms these findings. Altogether $60 \%$ of the GALT alleles have this mutation (and $58 \%$ of 117 patients studied when affected siblings were excluded), which is comparable to the findings of Elsas et al. (1993) of $66 \%$ on 52 patients. This contrasts with what was initially reported by Reichardt et al. (1991) of an overall frequency of only $26 \%$, which may have been due to a difference in the sampling procedure or a different study population.

However, the frequency of Q188R mutation among galactosemia patients is different if the presence or absence of GALT activity is taken into account. Previously, we had reported extensive biochemical heterogeneity among galactosemia patients identified either by newborn screenig or clinical recognition ( $\mathrm{Ng}$ et al. 1991). We have arbitrarily classified patients without detectable GALT activity as GG and those with detectable GALT activity ( $>0.1 \%$ of normal controls) as GV. Of the 132 patients who had DNA Q188R analysis, 113 were found to be GG and $19 \mathrm{GV}$. Of the GG group, a frequency of $67 \%$ of the GALT alleles for this mutation was obtained. This is in contrast to GV group with only $16 \%$. Of the latter group, not a single individual was a homozygote for the mutation. On the basis of this data, our preliminary conclusion is that patients homozygous for Q188R are devoid of GALT activity as measured by our multipoint assay. This finding indicates the importance of proper classification of galactosemia patients before results of either molecular studies or biochemical studies can be compared.

We have found that the frequency of Q188R differs among different racial and ethnic groups of patients. In the Caucasian group, of 95 patients, $64 \%$ of the alleles had this mutation. On the other hand, the frequency of Q188R mutation in the alleles among 12 African American patients was considerably lower (12\%). This is similar to the figure of $12.5 \%$ reported by Elsas et al. (1993) in a study of 4 patients.

The significantly lower frequency of Q188R in African-American galactosemia patients is noteworthy. It suggests that the African-American variant named previously as "Negro" variant of galactosemia (Segal 1969) is genetically distinct from the disease found in Caucasian patients. In fact, Reichardt et al. (1992) had proposed that the F171S mutation and S135L protein polymorphism were the result of racial admixture in African-American patients. The frequency of Q188R we observed in our study of African-American patients (12\%) approaches the frequency expected for Caucasian admixture: $0.64 \times 0.25$ $=0.16$ (Chakraborty et al. 1992). It also is noteworthy that Q188R is very rare in other racial groups such as Native American (Table 2), East Indian, Japanese, and Pakistani (J.K.V. Reichardt et al., unpublished). Thus, the molecular changes in galactosemia though present in all racial groups studied to date appear to vary considerably.

In this study of 132 patients, 19 were found to have detectable GALT activity, ranging from 0.02 to 1.1 units (normal controls of $\geq 20$ units). Since most of them were identified by newborn screening, it is not known how many would have had clinical problems if untreated. Cataracts was found in one patient in this study. Females including two adults with detectable GALT activity appear to maintain normal ovarian function (Kaufman et al. 1988). Whether the presence of minute GALT activity and normal levels of UDPGal in GV patients would preserve the ovarian function remains to be studied. A clinical correlation of cognitive, neurologic and ovarian outcome with the Q188R mutation in patients with absence of GALT activity is being reported (Kaufman et al. 1994).

Acknowledgement We thank many physicians who sent specimens to our laboratories for analysis, among them are Drs. Neil Buist, George Hogenson, Harvey L. Levy, and Charles Mize. We thank Maurice Peters (Dept. of Biochemistry and Molecular Biology, USC School of Medicine) for excellent technical assistance. The work in the laboratory of $\mathrm{W}$. $\mathrm{Ng}$ has been supported by NICHD grant HD $2640 \mathrm{I}$ and the work in the laboratory of J.K.V. Reichardt is supported by a Basil $0{ }^{\prime}$ Connor Starter Scholar Award from the March of Dimes (no. 5-FY 94-0824) and the Betty Lou Warren Research Fund. J.K.V. Reichardt is also a fellow of the James H. Zumberge Faculty Research and Innovation Fund at the University of Southern California.

\section{References}

Chacko DM, Christian JC, Nadler HL (1971) Unstable galactose1-phosphate uridyl transferase: a new variant of galactosemia. J Pediatr 78:454-460

Chacko CM, Wappner RS, Brandt IK., Nadler HL (1977) The Chicago variant of clinical galactosemia. Hum Genet 37: $261-279$

Chakraborty R, Kamboh M1, Nwankwo M, Ferrell RE (1992) Caucasian genes in American blacks: new data. Am J Hum Genet $50: 145-155$

Elsas LJ, Fridovich-Keil J, Hjelm N, Litwer S, Buist N, Tuerck J, Leslie ND, Holton J, Tyfield L (1992) Prognostication in galactosemia using molecular analysis. Am J Hum Genet 51 [Suppl]: A 167

Elsas II LJ, Friedovich-Keil JL, Leslie ND (1993) Galactosemia: a molecular approach to the enigma. Int Pediatr 8:101-109

Flach JE, Reichardt JKV, Elsas II LJ (1990) Sequence of a cDNA encoding human galactose- 1 -phosphate uridyl transferase Mol Biol Med 7:365-369

Hsia DY-Y, Walker FA (1961) Variability in the clinical manifestations of galactosemia. J Pediatr $59: 872-883$

Kaufman FR, Xu Yk, Ng WG, Donnel GN (1988) Correlation of ovarian function with galactose-1-phosphate uridyl transferase levels in galactosemia. J Pediatr 112:754-756

Kaufman FR, Reichardt JKV, Ng WG, Xu Y-K, Manis FR, McBride-Chang C. Wolff JA (1994), Correlation of aspects of cognitive. neurologic and ovarian outcome with the Q188R mutation of the galactose-1-phosphate uridyltransferase gene. $J$ Pediatr (in press) 
Leslie ND, Immerman EB, Flach JE, Florez M, Fridovich-Keil JL, Elsas LJ (1992) The human galactose-1-phosphate uridyltransferase gene. Genomics 14:474-480

Ng WG, Bergren WR, Donnell GN (1964) Galactose-1-phosphate uridyltransferase activity in galactosemia. Nature 203:845847

Ng WG, Xu Y-K, Kaufman FR, Donnell GN (1989) Deficit of uridine diphosphate galactose in galactosemia. J Inherit Metab Dis $12: 257-266$

Ng WG, Xu Y-K, Kaufman FR, Lee JES, Donnell GN (1991) Biochemical and clinical heterogeneity among 358 galactosemia patients. In: Wilcken B, Webster D (eds) Neonatal screening in the nineties. (8th International Neonatal Screening Symposium and Inaugural Meeting of the International Society of Neonatal Screening.) Leura, Australia, pp 181-188

Palmieri MJ, Berry GT, Playe DA, Roger S, Segal S (1991) The concentration of red blood cell UDPGlucose and UDPGalactose determined by high-performance liquid chromatography. Anal Biochem 194:388-393

Reichardt JKV (1992) Genetic basis of galactosemia. Hum Mut 1: 190-196

Reichardt JKV, Berg P (1988) Clonig and characterization of a cDNA encoding human galactose-1-phospate uridyl transferase. Mol Biol Med 5: 107-122

Reichardt JKV, Packman S, Woo SLC (1991) Molecular characterization of two galactosemia mutations: correlation of mutations and conserved domains in galactose-1-phosphate uridyl transferase. Am J Hum Genet 49:860-867
Reichardt JKV, Levy HL, Woo SLC (1992) Molecular characterization of two galactosemia mutations and one polymorphism: implication for structure-function analysis of human galactose1-phosphate uridyltransferase. Biochemistry 31:5430-5433

Schapira F, Kaplan J (1969) Electrophoretic abnormality of galactose-1-phosphate uridyl transferase in galactosemia. Biochem Biophys Res Commun 35:451-455

Segal S (1969) The Negro variant of congenital galactosemia. In: Hsia Y-Y (ed) Galactosemia. Thomas, Springfield, Ill, pp 176185

Segal S (1989) Disorder of galactose metabolism. In Scriver CR, Beaudet AL, Sly WS, Valle D (eds) The metabolic basis of inherited disease, 6th edn. McGraw-Hill, New York, pp 453-480

Segal S, Blair A, Roth H (1965) The metabolism of galactose by patients with congenital galactosemia. Am J Med 38:62-70

Waggoner DD, Buist NRM, Donnell GN (1990) Long-term prognosis in galactosemia: results of a survey of 350 cases. J Inherit Metab Dis 13:802-818

Xu YK, Ng WG, Giudici T, Kaufman FR, Donnell GN (1993) Further studies of uridine diphosphate galactose (UDPGal) deficiency in galactosemia (abstract). Soc Inherit Metab Dis, Pacific Grove, California, p 46 